\title{
Sternoclavicular Septic Arthritis Caused by Acupuncture over the Posterior Neck
}

\author{
Bang-Min Liu ${ }^{a}$, Tzong-Luen Wang ${ }^{a, b}$, Shih-Wen Hung \\ aEmergency Department, Shin-Kong Wu Ho-Su Memorial Hospital, Taipei, Taiwan

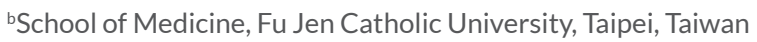

Received: 27/02/2015

Accepted: 06/03/2015

Published: 09/04/2015

How to cite this article: Liu M L, Wanga T L, Hung SW. Sternoclavicular septic arthritis caused by acupuncture over the posterior neck. EJCRIM 2015;2:doi: 10.12890/2015_000188

Conflicts of Interests: The authors declare that they have no conflicts of interest in this research

This article is licensed under a Commons Attribution Non-Commercial 4.0 License

\begin{abstract}
Acupuncture has been used therapeutically for thousands of years and is considered a relatively safe procedure. Sternoclavicular joint (SCJ) arthritis is a rare joint infection and has never been reported as an adverse event of acupuncture. We report the case of a 50-year-old woman who presented with progressive left neck, shoulder and upper chest pain after acupuncture. A computerized tomography (CT) scan revealed septic arthritis over the left sternoclavicular joint (SCJ) and methicillin-sensitive Staphylococcus aureus bacteraemia was noted. The patient was discharged uneventfully after intravenous antibiotic treatment. SCJ septic arthritis should be considered if unilateral neck and upper chest pain occurs after acupuncture.
\end{abstract}

\section{LEARNING POINTS}

- Sternoclavicular joint (SCJ) septic arthritis is rare but must be considered in any patient complaining of unilateral SCJ pain.

- Acupuncture over the neck area may result in SCJ septic arthritis.

- Infection control measures could minimize the number of adverse effects of acupuncture.

\section{KEYWORDS}

Sternoclavicular joint, septic arthritis, acupuncture

\section{INTRODUCTION}

Acupuncture has been used therapeutically in Asian countries for thousands of years and is growing in prominence in Europe and the United States. It is a relatively safe procedure, with rates of serious adverse effects estimated to be 0.05 per 10,000 treatments ${ }^{[1]}$. Most of the complications are physical injuries associated with acupuncture needles or problems related to wounds or systemic infection. The sternoclavicular joint (SCJ) is an unusual site of bacterial infection ${ }^{[2]}$ and SCJ septic arthritis has never been reported as a complication of acupuncture in the literature.

\section{CASE REPORT}

A 50-year-old woman with a history of type II diabetes mellitus and hepatitis B-related liver cirrhosis presented with severe left neck, shoulder and upper chest pain. According to her statement, she had become sore over her nuchal area 3 weeks previously and went to a local clinic for help. A muscle strain was diagnosed and she received an acupuncture procedure over the bilateral posterior neck. On the day following the procedure, left neck and shoulder pain developed and worsened despite several days of rest. The woman visited the clinic again and oral analgesics were prescribed without effect. Severe pain when rotating her left shoulder and swelling over the left upper chest were noted 2 days before she visited the emergency department. She denied fever, dysphagia and dyspnoea during the course of the condition. On physical examination, the patient looked ill with the following vital signs: body temperature: $36.2^{\circ} \mathrm{C}$, heart rate: 79 beats per minute, respiratory rate: 20 breaths per minute, blood pressure 130/78 $\mathrm{mmHg}$. Examination of the head, ears and eyes was normal and no cervical lymph nodes were palpated. Erythaema was noted over the left upper chest area with a swollen and tender sternoclavicular joint (Fig. 1). Chest pain worsened when the left shoulder was moved. Muscle power and sensation in the limbs were normal.

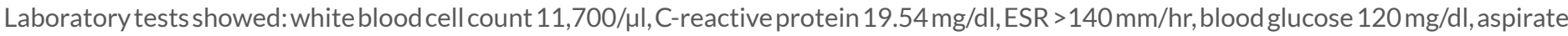
aminotransferase $19 \mathrm{U} / \mathrm{l}$ and creatinine $0.32 \mathrm{mg} / \mathrm{dl}$. A computerized tomography (CT) scan revealed oedema of the left sternoclavicular joint with anterior mediastinum extension (Fig. 2). Oxacillin and ceftriaxone were given after the CT scan. Blood culture revealed methicillinsensitive Staphylococcus aureus (MSSA) infection and antibiotics were changed to oxacillin plus gentamycin. The patient was discharged with complete recovery after 3-week intravenous antibiotic treatment. 

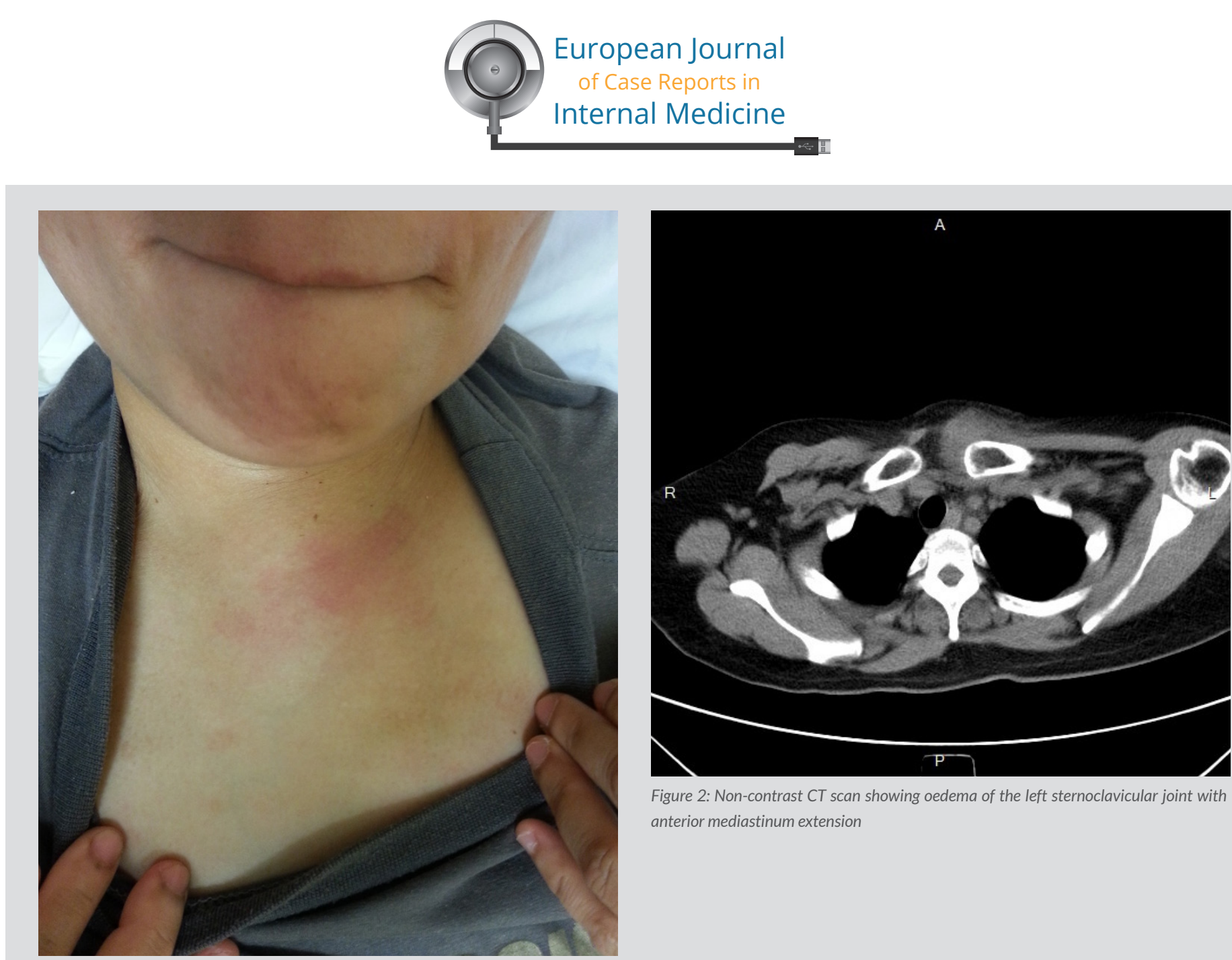

Figure 2: Non-contrast CT scan showing oedema of the left sternoclavicular joint with anterior mediastinum extension

Figure 1: Erythaema over the patient's left upper chest

\section{DISCUSSION}

Septic arthritis of the SCJ is rare and accounts for only $0.5-1 \%$ of all joint infections. It is often overlooked and has an insidious onset ${ }^{[2]}$. Common risk factors include intravenous drug use, infection at a distant site, diabetes mellitus, trauma, and an infected central venous line. It may spread from a distant source by haematogenous dissemination or from a nearby infection. SCJ septic arthritis is more prevalent in intravenous drug abusers (IVDA) than in the general population (17\% vs $1 \%)^{[3]}$, presumably because bacteria may enter the SCJ of IVDA from the adjacent internal jugular vein or subclavian vein if phelibitis or valvulitis of these veins is present. This hypothesis is consistent with the higher rate of bacteraemia in septic arthritis of the SCJ than of other joints (62\% vs 24\%). An infection must be considered in any patient complaining of unilateral SCJ pain until proven otherwise. MSSA is the most common pathogen ${ }^{[4]}$. An empiric antibiotic against methicillinresistant Staphylococcus aureus (MRSA) is indicated if the patient has risk factors such as intravenous drug use, haemodialysis, central venous access, recent hospitalization, or lives in an MRSA high-prevalence area. Surgical intervention may be needed if phlegmon of the chest wall or retrosternal abscess formation occurs.

Acupuncture is generally safe, and most of its major adverse events are associated with poorly trained unlicensed acupuncturists ${ }^{[1]}$. Adverse events include transmission of disease, pneumothorax, pneumoperitoneum, organ puncture, cardiac tamponade, local infection and bleeding ${ }^{[5]}$. The majority of adverse events are infections, which may be local or systemic. The acupuncture-related SCJ infection in our case may share a similar mechanism with such infections in IVDA. Bacteria might be introduced to the venous circulation in the neck through the insertion of non-sterile needles and cause SCJ septic arthritis. Infection control measures, such as the use of disposable needles, skin disinfection procedures and aseptic techniques, can minimize the number of adverse effects of acupuncture.

\section{CONCLUSION}

Acupuncture is regarded as a safe treatment but is not risk free. Safe and clean practice is necessary. SCJ septic arthritis should be considered if unilateral neck and upper chest pain occurs after acupuncture.

\section{REFERENCES}

Lao L, Hamilton GR, Fu J, Berman BM. Is acupuncture safe? A systematic review of case reports. Altern Ther Health Med 2003;9:72-83

Womack J. Septic arthritis of the sternoclavicular joint. J Am Board Fam Med 2012;25:908-12.

Ross JJ, Shamsuddin H. Sternoclavicular septic arthritis: review of 180 cases. Medicine (Baltimore) 2004;83:139-148.

El Ibrahimi A, Daoudi A, Boujraf S, Elmrini A, Boutayeb F. Sternoclavicular septic arthritis in a previously healthy patient: a case report and review of the literature. Int J Infect Dis 2009;13:e119-21.

Ernst E, White AR. Prospective studies of the safety of acupuncture: a systematic review. Am J Med 2001;110:481-5. 